\title{
L'origine de la lignée germinale et de la mortalité cellulaire
}

\author{
Herman Denis, Jean-Claude Lacroix
}

Chez beaucoup d'animaux, les cellules embryonnaires se répartissent en deux lots, un lot germinal et un lot somatique. Les cellules germinales sont potentiellement immortelles, puisqu'elles se perpétuent de génération en génération. En cela, elles different des cellules somatiques, qui sont mortelles, dans le sens où elles cessent de se multiplier après un certain nombre de divisions. Nous proposons une interprétation évolutive de la mortalité cellulaire, en supposant que cette propriété apparut chez les animaux les plus primitifs et fut un facteur crucial pour limiter l'expansion du soma, donc la taille de ces organismes rudimentaires.

Pour ceux-ci, la mortalité cellulaire était une innovation, donc un gain de fonctions par rapport à leurs ancêtres unicellulaires, qui étaient immortels. Les cellules mortelles devaient donc exprimer des fonctions supplémentaires par rapport aux cellules immortelles. Une telle différence paraît bien exister chez certains organismes actuels : plusieurs gènes ou produits de gènes doivent être inactivés pour que les cellules somatiques recouvrent l'immortalité.

\section{TIRÉS A PART}

H. Denis.

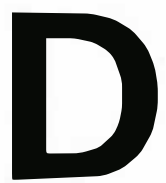
eux hypothèses principales ont été proposées pour expliquer la sénescence et la mort cellulaire. Une première hypothèse impute ces phénomènes à une accumulation progressive de défauts dans la machinerie cellulaire, dus à des mutations ou à des erreurs dans la synthèse de l'ARN et des protéines. Une seconde hypothèse considère la sénescence et la mort comme programmées génétiquement. Nous présentons ici une interprétation évolutive de la mortalité cellulaire qui prend résolument parti pour la seconde hypothèse. L'idée de base est que la mortalité apparut très tôt au cours de l'évolution des animaux. Dès le début, une ségrégation s'établit entre deux catégories de cellules, les unes immortelles, les autres mortelles. Une telle ségrégation s'est maintenue dans de nombreux groupes zoologiques. Elle s'instaure au cours du développement embryonnaire. Chez les animaux où la ségrégation se fait précocement, elle est en quelque sorte inscrite dans le cytoplasme de l'œuf, sous la forme d'un territoire qui fixe la destinée des cellules immortelles de l'embryon, puis de l'adulte.

\section{La théorie germinale}

Proposée il y a plus d'un siècle [1, 2], la théorie germinale postule l'existence chez les animaux de deux catégories distinctes de cellules. Les unes forment le germen et fourniront les gamètes. Les autres forment le soma ; elles édifieront tous les organes de l'adulte, y compris les gonades.
De fait, l'embryon de beaucoup d'animaux met précocement en réserve un lot de cellules germinales, appelées gonocytes. Dans de nombreux groupes, les gonocytes s'individualisent dès le début du développement [3, 4]. Ils peuvent même devenir repérables pendant que l'œuf se segmente. C'est le cas notamment pour les nématodes (Ascaris, Caenorhabditis) et les insectes (Miastor, Drosophila). Les gonocytes apparaissent toujours en nombre limité : quelquesuns chez les nématodes; quelques dizaines chez les amphibiens et les mammifères $[5,6]$. A ce moment, l'embryon comporte déjà plusieurs centaines à plusieurs milliers de cellules somatiques. Lorsque apparaissent les ébauches génitales, les gonocytes y pénètrent, se multiplient puis se différencient.

En général, le germen ne comprend que deux catégories de cellules, qui donneront naissance aux gamètes mâles et femelles. Ces cellules restent indépendantes et ne forment jamais un tissu cohérent parce qu'elles n'adhèrent pas les unes aux autres. Le soma se diversifie en de nombreux types cellulaires. Chez les animaux les plus simples, il s'organise en deux feuillets : l'ectoderme et l'endoderme. Les animaux plus évolués comportent un feuillet supplémentaire : le mésoderme. Ils sont donc tridermiques. Il est vraisemblable que les animaux tridermiques dérivent d'un ancêtre didermique, et que l'acquisition d'un troisième feuillet s'est faite précocement au cours de l'évolution des métazoaires [7]. 


\section{La dichotomie germen-soma}

La théorie germinale est-elle applicable à tous les êtres vivants ? La dichotomie germen-soma n'est pas toujours aussi nette que chez les nématodes et les insectes. Dans de nombreux groupes animaux, le germen ne devient détectable qu'à un stade avancé de l'ontogenèse. Chez les végétaux, il est indistinct. Comment expliquer ces différences? On peut admettre que plusieurs événements évolutifs ont créé les lignées conduisant aux principaux types d'organisation connus à l'heure actuelle (moisissures, plantes, animaux...). La ségrégation entre germen et soma ne serait apparue que dans la lignée animale. On peut aussi considérer les organismes multicellulaires comme monophylétiques, c'està-dire issus d'un ancêtre commun. Dans ce cas, il convient de se demander si l'absence de germen reconnaissable est un caractère primitif ou secondaire. Pour se faire une opinion, il faut examiner comment se reproduisent les principaux groupes d'êtres vivants.

Bien qu'immortels, les protozoaires ciliés tels que la paramécie montrent une ébauche de spécialisation germinale et somatique [8]. Ces cellules possèdent deux types de noyaux : le micronucleus et le macronucleus. Le micronucleus transmet les gènes de génération en génération et subit la méiose avant la conjugaison. Il est donc assimilable au germen. Le macronucleus se forme à partir du micronucleus. Il multiplie les gènes qu'il contient et dirige les fonctions cellulaires durant l'intervalle entre deux conjugaisons, puis disparaît. Le macronucleus peut donc être comparé au soma.

La dualité germen-soma se retrouve chez les protistes coloniaux tels que Volvox carteri. Cet organisme ne comporte que deux catégories de cellules, correspondant au germen et au soma des animaux. Les cellules germinales de $V$. carteri sont immortelles. Elles seules ont la capacité de subir la méiose. Les cellules somatiques remplissent les fonctions végétatives, telles que la locomotion et la phototaxie.

de fois, après quoi elles dégénèrent et meurent [9].

On observe une ségrégation précoce entre germen et soma dans quinze phylums (embranchements) d'animaux didermiques et tridermiques, parmi lesquels figurent les cténaires, les nématodes, les mollusques, les arthropodes et les vertébrés [10]. Douze phylums ne manifestent qu'une ségrégation tardive ou aucune ségrégation [10]. Il paraît peu vraisemblable que la dichotomie germensoma soit apparue à de multiples reprises au cours de l'évolution. Il est plus simple de supposer qu'une telle dichotomie existait déjà chez l'ancêtre commun de tous les animaux, et peut-être même chez celui de tous les organismes multicellulaires.

\section{Reproduction sexuée et asexuée}

De nombreuses lignées animales ont conservé l'aptitude que nous considérons comme primitive à séparer le germen du soma dès les premiers stades de l'ontogenèse. D'autres lignées ont progressivement retardé la ségrégation, tout en conservant un germen identifiable. Chez la souris, par exemple, les gonocytes ne deviennent repérables que vers le septième jour de développement [5]. Ce retard est sans doute lié à la mise en place très précoce du trophectoderme, nécessaire à la formation du placenta.

Il existe des groupes d'animaux où la ségrégation germen-soma est tellement tardive que toute distinction s'estompe entre les deux lignées cellulaires [3]. Cette tendance évolutive présente des avantages : les cellules somatiques gardent intacte la forte capacité de prolifération qui caractérise les cellules germinales. Cela favorise la régénération et la reproduction asexuée, qui consistent l'une et l'autre en une prolifération de masses cellulaires d'origines embryologiques diverses. De fait, la reproduction asexuée ne s'observe que dans les groupes zoologiques où le germen reste indistinct ou se sépare tardivement $[3,10]$. Elle est particulièrement fréquente chez les animaux didermiques $[3,10]$.

La reproduction asexuée se déroule suivant des modalités très variables (bourgeonnement, scissiparité...). On décèle une plus grande uniformité dans la reproduction sexuée, et en particulier dans le processus fondamental qui la conditionne : la méiose. C'est un argument de poids pour considérer la reproduction sexuée comme primitive par rapport à la reproduction asexuée [3]. Apparue plus tardivement, la reproduction asexuée aurait fait disparaître chez de nombreux organismes toute ségrégation nette entre germen et soma. L'absence de germen reconnaissable serait donc une conséquence de l'adoption par certains groupes animaux de deux modes de propagation somatique : la régénération et la reproduction asexuée.

\section{Apparition de la mortalité cellulaire}

Il est très probable que les premiers organismes pluricellulaires se formèrent à partir de cellules isolées qui étaient immortelles et se reproduisaient de façon sexuée. On peut imaginer que ces organismes très primitifs étaient organisés comme Volvox. Pour eux, l'une des innovations principales fut l'acquisition de la mortalité cellulaire, que nous définissons comme l'aptitude des cellules somatiques à subir un nombre limité de divisions. De la sorte, l'expansion du soma se trouvait automatiquement restreinte. Cette limitation était cruciale car un organisme de trop grande taille n'aurait pas été viable : il n'existait pas de système qui pût distribuer aux cellules l'oxygène et les nutriments, ni éliminer leurs déchets.

Au début, le soma avait comme fonctions de protéger et de nourrir le germen. De ce fait, il contrôlait la prolifération des cellules germinales, parce que celles-ci dépendaient de facteurs trophiques pour se diviser, comme leurs ancêtres unicellulaires. De ces derniers, les cellules germinales reçurent en héritage d'autres propriétés essentielles : l'immortalité, la non-adhérence entre congénères et l'aptitude à réaliser la méiose.

\section{Contrôle de la prolifération et de la mort cellulaires}

L'acquisition de la mortalité cellulaire était de nature à limiter la taille du soma chez les premiers organismes Elles se divisent un certain nombre 
pluricellulaires. Les choses semblent encore fonctionner de cette manière chez certaines volvocales. Un contrôle aussi grossier de la prolifération cellulaire était insuffisant pour permettre au soma de se diversifier en organes de plus en plus complexes. Il fallait aussi maîtriser la multiplication des produits sexuels autrement que par un simple apport de nutriments. Un contrôle plus fin s'est progressivement établi grâce à un circuit de régulation positive et négative, qui s'est instauré aussi bien dans la lignée germinale que dans la lignée somatique.

Parallèlement s'est mis en place un contrôle de la pérennité cellulaire, qui décide si une cellule doit continuer à vivre ou mourir prématurément, en commettant une sorte de suicide, appelé apoptose [11]. La mort par apoptose peut être assimilée à une mitose abortive [11]. Elle diffère profondément de la mort passive, qui exprime une propriété intrinsèque des cellules somatiques, et survient plus tard dans la vie de l'organisme.

Dans la lignée germinale comme dans la lignée somatique, les divisions cellulaires sont déclenchées par des agents extérieurs, appelés facteurs de croissance. Ces facteurs émanent d'autres cellules. De même, le maintien en vie des cellules dépendrait de facteurs extra-cellulaires [12]. Dans l'embryon, ce double système détermine la taille et la forme de l'organisme en devenir, car le modelage des différentes ébauches dépend non seulement de la multiplication des cellules, mais aussi de la mort de certaines d'entre elles [12]. Chez l'adulte, les facteurs de croissance conditionnent le renouvellement des cellules, ainsi que la prolifération des gamètes, tandis que d'autres facteurs entraînent l'élimination par apoptose des cellules indésirables [12].

On comprend mieux le mode d'action des facteurs de croissance que celui des facteurs déterminant la survie cellulaire $[12,13]$. La transmission des signaux de croissance est impliquée dans la transformation tumorale. Les proto-oncogènes ou gènes du cancer spécifient soit les facteurs de croissance eux-mêmes, soit d'autres protéines intervenant dans l'échange des signaux véhiculés par les facteurs de croissance. Les pro$m / s n^{\circ} 7$, vol. 8, seplembre 92 duits des proto-oncogènes stimulent la division cellulaire. D'autres gènes, appelés suppresseurs de tumeurs ou anti-oncogènes, ont une action antagoniste : leur fonction est de ralentir ou d'arrêter la prolifération des cellules. Parmi les suppresseurs de tumeurs, les mieux connus sont le gène spécifiant la protéine p53 et le gène du rétinoblastome (Rb) [14]. Dans les cellules tumorales, le mécanisme gouvernant la prolifération cellulaire est perturbé : un nombre variable de proto-oncogènes est muté ou amplifié, tandis que les supresseurs de tumeurs sont fréquemment délétés ou inactivés.

\section{Contrôle du cycle cellulaire}

Tout le système contrôlant la prolifération des cellules commande deux événements clés du cycle cellulaire : le démarrage de la réplication de l'ADN (restriction G1/S), et l'entrée en mitose (transition G2/M). Le contrôle de l'entrée en mitose commence à être compris [15]. Un rôle essentiel est joué par le facteur MPF (maturation promoting factor). Celui-ci est un hétérodimère formé de deux molécules: une cycline et une protéine kinase appelée p34 cdc2. Une autre cycline interviendrait dans le franchissement du seuil G1/S [16]. Les suppresseurs de tumeurs agissent directement sur le cycle cellulaire : p53 inhiberait la réplication de l'ADN [17], tandis que le produit de $\mathrm{Rb}$ inhiberait la synthèse de p34 ${ }^{\text {cdc2 }}$, bloquant ainsi la transition G2/M [18].

Les cellules somatiques et les cellules germinales partagent plusieurs éléments de la chaîne qui gouverne la prolifération cellulaire. Certains de ces éléments déterminent à la fois l'entrée en mitose et l'entrée en méiose. Par exemple, le MPF, qui déclenche la mitose dans les cellules somatiques, a été initialement défini comme un facteur commandant la reprise de la méiose dans l'oocyte des amphibiens [15]. Parmi les protooncogènes connus, beaucoup sont actifs dans les cellules germinales [19]. C'est notamment le cas pour le proto-oncogène mos: le produit de mos a pu être identifié avec le facteur cytostatique [20], qui bloque l'oocyte en métaphase de la seconde division méiotique et interagit avec le
MPF [6]. Au moins un gène suppresseur de tumeurs (p53) est exprimé dans l'oocyte des amphibiens, mais sa fonction $y$ reste inconnue [21].

Beaucoup de choses restent à découvrir concernant les processus qui déclenchent l'entrée en mitose des cellules somatiques, ainsi que la prolifération puis l'entrée en méiose des gonocytes. En ce qui concerne l'évolution de ces systèmes, on sait que les organismes unicellulaires et les animaux utilisent, pour déclencher la mitose et la méiose, des mécanismes très similaires, puisqu'un élément commun aux deux systèmes ( $\left.\mathrm{p} 34^{\mathrm{cdc} 2}\right)$ est interchangeable entre les cellules humaines et celles de levure [15]. Manifestement, la commande directe d'entrée en mitose et en méiose n'a guère évolué depuis l'origine des organismes pluricellulaires. C'est en amont qu'est apparue la nouveauté. Dans ce cas comme dans beaucoup d'autres, il ne s'agit pas nécessairement d'une création ex nihilo de nouveaux mécanismes, mais. plutôt d'un réemploi de mécanismes plus anciens, donc de gènes préexistants [22].

Ainsi, la chaîne de transmission des signaux mitotiques pourrait dériver d'une chaîne similaire qui contrôlait la conjugaison chez les prédécesseurs des métazoaires. Pour se conjuger, les protozoaires doivent se rencontrer, s'unir, puis déclencher la division des noyaux dans chacun des partenaires $[3,8]$. Cela implique l'échange de signaux extracellulaires, donc le recours à des récepteurs membranaires [23] et à un appareil de transduction, qui fait probablement intervenir certains proto-oncogènes ubiquitaires tels que ras [24]. Ce dernier est utilisé par les animaux pour un autre usage : la transduction des signaux mitotiques. Si notre hypothèse est juste, il devrait exister une homologie entre les facteurs d'appariement (gamones) échangés par les protozoaires et les facteurs de croissance échangés par les cellules animales. C'est peut-être le cas pour une interleukine de mammifère, qui ressemble à une gamone d'Euplotes [23].

\section{Acquisition} de la mortalité cellulaire

Au cours de l'évolution, la mise en 
place de processus complexes gouvernant la division et la survie des cellules n'a pas effacé une innovation beaucoup plus ancienne : la mortalité passive ou intrinsèque, que nous opposons à la mort active (apoptose). Dans l'apparition des fonctions régissant la prolifération et la mort cellulaires, nous distinguons trois acquisitions successives: mortalité passive, contrôle de la mitose, puis de l'apoptose par des facteurs extra-cellulaires. Chez les animaux supérieurs, la mortalité passive conditionne la longévité des différentes espèces [25]. Quand elles ont accompli le nombre de divisions qui leur est imparti, les cellules subissent des modifications caractéristiques, connues sous le nom de sénescence. Les cellules germinales échappent à cette fatalité : elles sont immortelles, tout au moins potentiellement, puisqu'elles se perpétuent de génération en génération.

Avant que la différenciation ne débute, les cellules embryonnaires sont apparemment immortelles : mises en culture, elles semblent capables de proliférer à l'infini [25]. Réimplantées dans un embryon, elles peuvent s'intégrer à la lignée germinale [26]. Le caractère mortel n'apparaît que plus tard dans le développement (figure 1). Ces expériences n'ont été réalisées que chez les mammifères, où la séparation entre le germen et le soma est assez tardive [5]. Dans les groupes où le germen s'individualise précocement, le caractère mortel pourrait s'instaurer dès le début de l'ontogenèse. Quoi qu'il en soit, la chronologie des événements embryonnaires semble bien refléter celle des événements évolutifs. Dans l'embryon, le caractère immortel précède le caractère mortel (figure 1). Au cours de l'évolution, l'immortalité a précédé la mortalité.

Suivant notre schéma évolutif, la mortalité fut une innovation pour les premiers organismes pluricellulaires, donc un gain de fonctions par rapport à leurs prédécesseurs. Si cette idée est correcte, les cellules mortelles devaient exprimer par rapport aux cellules immortelles une ou plusieurs fonctions supplémentaires, qui se sont ajoutées à la chaîne préexistante de contrôle de la division cellulaire. Une telle différence s'est-elle maintenue chez les organismes actuels?

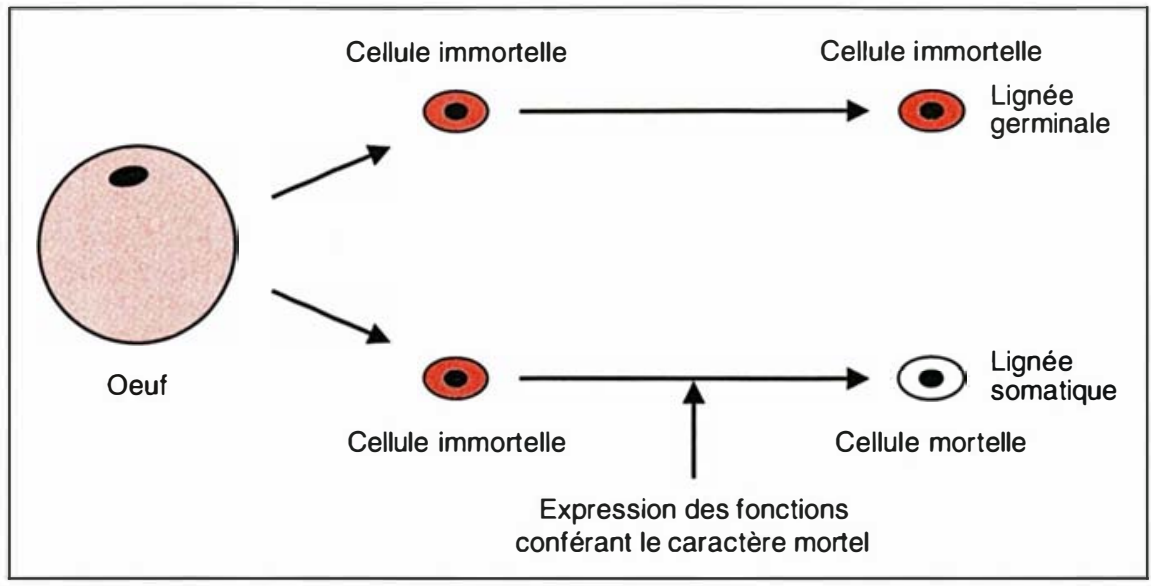

Figure 1. Acquisition du caractère mortel par les cellules embryonnaires. On ne connaît pas bien le stade où s'instaure la mortalité cellulaire au cours du développement, ni les facteurs qui entraînent son apparition. Ces facteurs provoquent à terme la sénescence des cellules somatiques. D'autres facteurs agissent plus précocement pour déclencher l'apoptose, c'est-à-dire la mort prématurée de certaines cellules. Les cellules germinales sont protégées à la fois contre la sénescence et contre l'apoptose.

Chez Volvox, il existe au moins un gène $(\operatorname{reg} A)$ dont le produit empêche les cellules somatiques de devenir reproductrices, donc immortelles [27]. Chez les animaux, aucune comparaison directe n'a été faite entre les cellules somatiques et les cellules germinales pour ce qui concerne les fonctions gouvernant la mortalité et l'immortalité. En revanche, de nombreux travaux ont été consacrés aux cellules immortalisées, qui se multiplient indéfiniment en culture. En effet, l'immortalisation est un facteur de risque dans la voie conduisant à la transformation cancéreuse, sans toutefois être indispensable pour que la conversion ait lieu [28].

Si l'on fusionne des cellules immortalisées d'origine humaine avec des fibroblastes normaux, on crée des hybrides dont le nombre de divisions sera limité (figure 2, [29]). Le phénotype mortel est donc dominant par rapport au phénotype immortel. Cette observation peut s'interpréter simplement : le fibroblaste apporte à l'hybride des fonctions - qui faisaient défaut à la cellule immortalisée - parce que les gènes correspondants y sont inactivés.

\section{Causes de \\ l'immortalisation cellulaire}

Les cellules somatiques sont bien protégées contre l'immortalisation. Chez l'homme, par exemple, les fibroblastes ne s'immortalisent pas spontanément [25]. Les choses sont différentes pour les rongeurs, où la fréquence d'immortalisation est faible, mais mesurable [25]. Selon certains auteurs, deux types d'obstacles doivent être surmontés pour que les cellules humaines deviennent immortelles [30]. Au total, ces obstacles mettraient en jeu au moins quatre groupes de complémentation [31]. Le modèle d'immortalisation en deux étapes découle d'études réalisées sur des cellules en culture [30].

L'arrêt au premier obstacle (M1) se produit lorsque les cellules cessent de répondre aux facteurs de croissance et de répliquer leur $\mathrm{ADN}$. Elles entrent alors en sénescence. Le blocage peut être levé par des protéines spécifiées par les virus transformants, telles que l'antigène $\mathrm{T}$ de SV40. Ce dernier se lie aux protéines $\mathrm{Rb}$ et $\mathrm{p} 53$, qui sont des inhibiteurs de prolifération, et les inactive $[14,30]$. Les cellules recom- 

que la machinerie de réplication était intacte et prête à repartir. Après 20-30 divisions, un nouveau blocage s'instaure. La population cellulaire cesse de croître, puis diminue parce que le nombre de morts l'emporte peu à peu sur le nombre de divisions. Cette période est appelée crise. Très mencent à se diviser, ce qui montre

peu de cellules surmontent ce second obstacle (M2). Pour qu'un tel événement ait lieu, il faut inactiver un ou plusieurs gènes non encore identifiés [30, 31]. On obtient ainsi des cellules immortelles, qui requièrent cependant pour proliférer la présence de l'antigène $T$ ou d'une protéine de même fonction. En effet, le premier

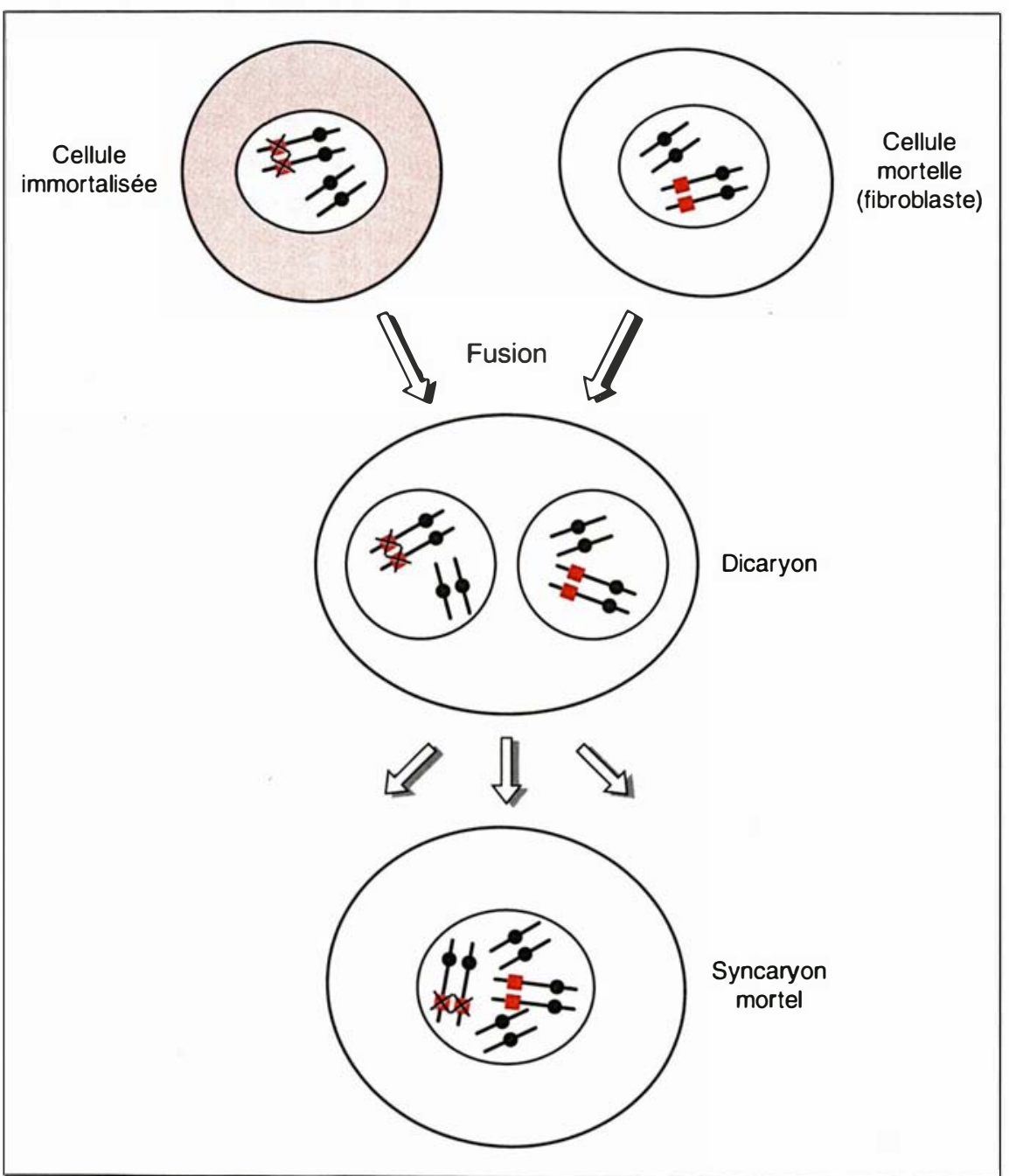

Figure 2. Expérience de fusion entre un fibroblaste humain et une cellule immortalisée de même origine. La fusion produit un hybride possédant deux noyaux (dicaryon). Après un certain nombre de divisions, les noyaux fusionnent à leur tour. Les cellules mononucléées ainsi obtenues (syncaryons) sont mortelles. On interprète ce résultat en supposant que la cellule mortelle exprime des fonctions qui sont perdues dans la cellule immortalisée parce que les gènes correspondants sont inactivés par délétion ou par mutation. Une de ces mutations est représentée sur la figure par un carré rouge barré d'une croix noire. Elle affecte les deux chromosomes d'une même paire. Cela traduit le caractère récessif des mutations touchant les cellules immortalisées. Si ces mutations étaient dominantes, la cellule immortalisée imposerait son caractère à l'hybride. On notera cependant que certaines cellules immortelles d'origine tumorale peuvent former avec des cellules normales des hybrides immortels, comme les hybridomes. Dans ce cas, au moins un des proto-oncogènes de la cellule tumorale porte une mutation dominante.

$\mathrm{m} / \mathrm{s} n^{\circ} 7$, vol. 8 , septembre 92 obstacle à la prolifération (M1) subsiste toujours [30]. L'antigène $T$ ne fait que supprimer ses effets.

Jusqu'à présent, les études d'immortalisation cellulaire ont surtout porté sur l'homme et les rongeurs. Il serait intéressant de savoir si tous les grands groupes zoologiques possèdent les deux mécanismes (M1 et M2) qui font obstacle à l'immortalisation, et qui sont causes de mortalité. Cela permettrait de préciser lequel parmi ces deux mécanismes est apparu le premier au cours de l'évolution. En tous cas, il semble bien que le mécanisme M2 soit absent chez les rongeurs, ce qui expliquerait pourquoi les cellules de ces animaux s'immortalisent assez fréquemment [32].

C'est donc bien une perte de fonction qui engage les cellules dans la voie de l'immortalité. Dans les cas pathologiques, la perte de fonction est causée soit par mutation, soit par inactivation du produit des gènes qui confèrent le phénotype mortel. Dans l'embryon, le caractère immortel se maintiendrait en quelque sorte par défaut, parce que les gènes de mortalité demeurent inactifs (figure 1). Cependant, il est possible que les cellules germinales possèdent un mécanisme empêchant l'apparition du caractère mortel. L'immortalité, caractère ancestral hérité des organismes unicellulaires, aurait donc subsisté chez les animaux grâce à l'acquisition de deux fonctions antagonistes : une fonction de mortalité exprimée dans les cellules somatiques et une fonction supprimant la mortalité, exprimée dans les cellules germinales.

\section{Nature des mutations engendrant l'immortalité}

En quoi consiste le second obstacle (M2) empêchant la prolifération indéfinie des cellules humaines? Un des gènes impliqués pourrait intervenir dans le contrôle de la réplication des télomères [33]. Normalement, l'ADN chromosomique se raccourcit à chaque cycle de réplication (figure 3, p. 700). A terme, les chromosomes perdent leurs télomères et deviennent instables : ils ont tendance à fusionner, formant ainsi des cercles, des dicentriques, etc. [34]. Le processus est lent à cause de la structure même 
des télomères. Chacun d'eux comporte un motif simple de quelques paires de bases, répété à l'identique plusieurs dizaines à plusieurs centaines de fois [34]. Plus longues sont les séquences télomériques, plus grand est le nombre de divisions qu'une cellule peut subir avant de souffrir d'instabilité chromosomique, puis de mourir.

Les cellules germinales humaines ainsi que les cellules immortalisées sont apparemment protégées contre l'érosion de leurs chromosomes [35]. Elles possèdent une enzyme particulière, appelée télomérase, qui ajoute des unités de répétition à l'extrémité des chromosomes, évitant ainsi leur raccourcissement [34]. La télomérase comporte une partie protéique et une partie ribonucléique, qui contient l'information nécessaire pour la synthèse des unités télomériques. La télomérase est donc une rétrotranscriptase capable de copier en ADN une partie de sa propre séquence [34].

Comment concilier cette hypothèse avec le modèle évolutif que nous proposons? Les protozoaires possèdent une télomérase, comme les cellules animales [34]. Il est logique de penser que les ancêtres unicellulaires des animaux actuels disposaient déjà d'une enzyme de même spécificité. Dans certaines lignées animales, les cellules somatiques développèrent un système apte à réprimer la synthèse de la télomérase, auparavant constitutive. Ce gain de fonction recquiert l'acquisition de nouveaux gènes. Ces gènes pourraient appartenir à la famille des suppresseurs de tumeurs, puisqu'ils ont comme fonction d'arrêter la prolifération cellulaire.

Chez les espèces qui possèdent le mécanisme M2, le système réprimant la synthèse de la télomérase se mettrait en place au cours du développement embryonnaire (figure 1). L'inhibition ne s'instaure pas dans les cellules germinales, qui peuvent se multiplier indéfiniment sans que leurs chromosomes se raccourcissent. Cette absence d'inhibition pourrait être un phénomène actif plutôt que passif : les cellules germinales disposeraient d'un mécanisme apte à inhiber la mise en fonction du système réprimant la production ou l'activité de la télomérase.

Pour ce qui concerne les cellules immortalisées d'origine humaine, nous supposons qu'elles ont perdu par mutation ou délétion un élément du mécanisme réprimant la synthèse

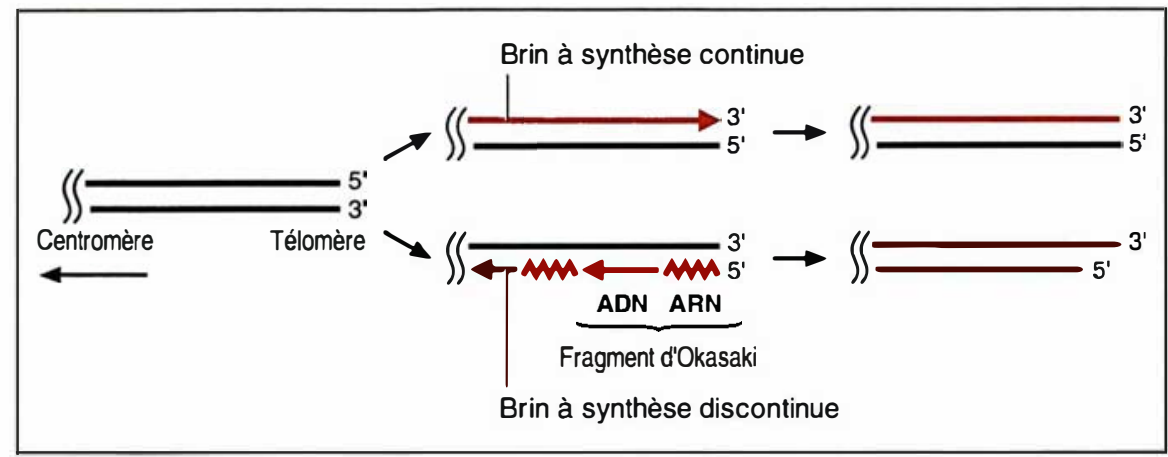

Figure 3. Raccourcissement de l'extrémité des chromosomes au cours de la réplication. A chaque télomère, un brin de I'ADN se réplique de façon continue, tandis que l'autre se réplique de facon discontinue. Sur le brin à synthèse discontinue, la réplication se fait par fragments d'environ 200 bases, dits fragments d'Okasaki, eux-mêmes amorcés par un court fragment d'ARN. Celui-ci est ensuite détruit et remplacé par un fragment d'ADN, copié sur le brin complémentaire. Le remplacement n'a lieu qu'entre les fragments d'Okasaki internes, et non à l'extrémité de l'ADN, parce que l'ADN polymérase ne peut qu'allonger des molécules déjà existantes, et non amorcer la synthèse d'une nouvelle chaîne. Par conséquent, les chromosomes se raccourcissent par leurs deux bouts à chaque réplication. Ils finissent par perdre leurs télomères, ce qui les rend instables. Les cellules immortelles doivent empêcher l'érosion de leurs chromosomes. Elles possèdent une enzyme capable de remplir cette fonction : la télomérase. ou l'activité de la télomérase. Cette perte ferait sauter le second obstacle (M2) sur la voie qui conduit à l'immortalisation, suivant le modèle présenté plus haut [30]. Le répresseur disparu, la synthèse de la télomérase pourrait se faire, ce qui instaure l'immortalité. Inversement, la mortalité réapparaît après fusion d'une cellule immortalisée avec une cellule mortelle (figure 2), parce que les chromosomes de celle-ci dirigent la synthèse d'une substance diffusible, qui arrête la production ou l'activité de la télomérase dans l'hybride.

Comme nous l'avons vu, les cellules des rongeurs s'immortalisent assez facilement. Il faut rapprocher cette observation d'une autre qui concerne l'érosion des télomères chez la souris. Un tel phénomène ne semble pas se produire dans les cellules somatiques de cet animal [34]. Tout cela s'explique si l'on admet que les rongeurs ne possèdent pas le mécanisme M2 évoqué précédemment, et que la télomérase intervient dans ce mécanisme. Les cellules de rongeur s'immortaliseraient aisément parce la télomérase y est exprimée de façon constitutive. Il ne serait donc pas nécessaire de la remettre en fonction, comme dans les cellules humaines.

\section{Les déterminants germinaux}

Dans l'embryon en voie de développement, les gonocytes peuvent souvent être reconnus grâce à des propriétés particulières de leur cytoplasme. Chez les mammifères, il s'agit d'une teneur élevée en phosphatase alcaline, repérable cytochimiquement [5]. Dans beaucoup de cas, les gonocytes contiennent des granulations typiques, opaques aux électrons. De telles granulations ont été identifiées dans des groupes aussi divers que les nématodes, les insectes, les amphibiens et les mammifères [6]. Quand les gonocytes se forment précocement, les granules germinaux peuvent être repérés dans l'œuf et même dans l'oocyte. Ainsi se trouve délimité un territoire ovulaire, appelé plasme germinal, que la segmentation de l'œuf répartit entre un petit nombre de blastomères. Parmi les cellules issues de ces derniers, seules deviendront gonocytes celles qui 
auront inclus une partie du plasme germinal. Dans l'œuf des amphibiens anoures, le plasme se trouve au pôle végétatif (pigmenté), aux confins du territoire endodermique présomptif. Dans l'œuf des insectes, il est localisé au pôle postérieur.

Deux questions fondamentales se posent à propos du plasme germinal. Premièrement, ce plasme peut-il être identifié avec les déterminants germinaux : un blastomère devient-il nécessairement gonocyte s'il inclut une partie du plasme germinal ? Deuxièmement, quelle est la nature des déterminants germinaux ? On peut répondre à la première question par l'affirmative. Chez la drosophile, on a injecté, au pôle antérieur de l'embryon, du cytoplasme provenant du pôle postérieur : des gonocytes fonctionnels se forment à l'endroit de l'injection [36].

En ce qui concerne la nature des déterminants germinaux, il semble que des ARN messagers particuliers, associés aux granules, jouent un rôle important [6, 37]. Des expériences déjà anciennes ont imposé l'idée d'une implication de l'ARN dans la détermination de la lignée germinale. Si l'on irradie par un faisceau de lumière ultraviolette le pôle végétatif d'un œuf d'amphibien anoure, on obtiendra une larve appauvrie en gonocytes, voire un adulte stérile [38]. Or c'est l'ARN que détruisent préférentiellement les rayons ultraviolets. Des expériences de même nature ont été réalisées sur des embryons de drosophile, avec des résultats identiques : l'irradiation du pôle postérieur de l'œuf empêche la formation des gonocytes [6]. Ceux-ci réapparaissent si l'on injecte du cytoplasme provenant du pôle postérieur d'œufs non traités. En purifiant les extraits cytoplasmiques, on a pu isoler une fraction contenant les ARN messagers, capable de restaurer partiellement les propriétés du plasme germinal irradié [39]. Parmi les messagers présents dans le plasme germinal de la drosophile, on trouve les produits des gènes oskar et nanos [37]. La séquence des protéines spécifiées par ces messagers est connue, mais leur mode d'action reste mal compris [40].

L'ARN n'est pas le seul constituant essentiel du plasme germinal. Des protéines jouent aussi un rôle capital, comme les produits des gènes vasa et staufen [37]. Les déterminants germinaux sont donc composites. Tous ces éléments sont produits durant l'oogenèse par des gènes à effet maternel [40]. Chez la drosophile, au moins sept gènes gouvernent la synthèse, la localisation et le maintien des composants du plasme germinal au pôle postérieur de l'oocyte [37, 40]. Parmi ces gènes, seul vasa spécifie un produit apparenté à une protéine de fonction connue : le facteur de traduction eIF-4A [41, 42].

\section{Conclusion}

Au cours du développement, certaines cellules embryonnaires deviennent somatiques, donc mortelles. D'autres deviennent germinales, donc potentiellement immortelles (figure 1). Le pouvoir de conférer l'immortalité est lié à des facteurs localisés dans le cytoplasme ovulaire. Il apparaît pendant l'oogenèse comme le fruit de l'activité de gènes à effet maternel. Dans l'œuf de beaucoup d'animaux, cette propriété n'est pas répartie uniformément. Elle est confinée à la région du cytoplasme qui contient les déterminants germinaux. Quand ce territoire est détruit, toutes les cellules issues de l'œuf deviennent somatiques.

Quel rôle assigner aux déterminants germinaux? On peut envisager qu'ils déclenchent une chaîne d'événements destinés à empêcher certaines cellules embryonnaires de devenir somatiques. $\mathrm{Si}$ cette interprétation est exacte, une des fonctions des déterminants germinaux serait d'inhiber directement ou indirectement l'expression des gènes qui confèrent le caractère mortel au soma. Cette inhibition aurait comme résultat d'éviter l'apparition des mécanismes qui freinent la prolifération des cellules somatiques et font obstacle à l'immortalisation

H. Denis, professeur à l'université Pierre-et-MarieCurie. Centre de génétique moléculaire Cnrs, avenue de la Terrasse, 91198 Gif-sur-Yvette Cedex, France. J.-C. Lacroix, professeur d̀ l'université Pierre-et-Marie-Curie. Laboratoire de génétique du développement, 7, quai SaintBernard, 75230 Paris Cedex 05, France.

\section{RÉFÉRENCES}

1. Nussbaum M. Die Differenzierung des Geschlechts im Thierreich. Arch Mikr Anat $1888: 18: 1-121$

2. Weismann A. Das Keimplasma. Eine Theorie der Vererbung. Jena: Fischer, 1892. 3. Brien P. Biologie de la reproduction animale. Paris : Masson, 1966.

4. Davidson EH. Gene Activity in Early Development. Orlando : Academic Press, 1986.

5. Ginsburg M, Snow MHL, McLaren A. Primordial germ cells of the mouse embryo during gastrulation. Development $1990 ; 110$ : 521-8.

6. Signoret J, Collenot A. L'organisme en développement. Des gamètes à l'embryon. Paris : Hermann, 1991.

7. Christen R, Ratto A, Baroin A, Perasso R, Grell KG, Adoutte A. An analysis of the origin of metazoans, using comparisons of partial sequences of the $28 \mathrm{~S}$ RNA, reveals an carly emergence of triploblasts. EMBO J 1991; 10 : 499-503.

8. Sonneborn TM. Heredity, development and evolution in Paramecium. Nature 1955 ; 175 : 1100-6.

9. Mignot JP. Les cœnobes chez les volvocales : un exemple du passage des unicellulaires aux pluricellulaires. Ann Biol 1985 ; 24: 1-26.

10. Buss LW. Evolution, development, and the units of selection. Proc Nall Acad Sci USA $1983 ; 80: 1387-91$.

11. Ucker DS. Death by suicide : one way to go in mammalian cellular development ? New Biol 1991; 3 : 103-9.

12. Raff MC. Social controls of ccll survival and cell death. Nature $1992 ; 356$ : 397-400.

13. Cross M, Dexter TM. Growth factors in development, transformation, and tumorigenesis. Cell 1991; 64: 271-80.

14. Levine AJ. Tumor suppressor genes. BioEssays $1990 ; 12: 60-6$.

15. Nurse P. Universal control mechanism regulating onset of $\mathrm{M}$-phase. Nature 1990 ; 344 : 503-8.

16. Girard E, Strausfeld V, Fernandez A, Lamb NJC. Cyclin A is required for the onset of DNA replication in mammalian fibroblasts. Cell 1991; 67 : 1169-79.

17. Marshall CJ. Tumor suppressor genes. Cell 1991; 64: 313-26.

18. Dalton S. Cell cycle regulation of the human $c d c 2$ gene. EMBO J $1992 ; 11$ : 1797-804.

19. Propst F, Rosenberg MP, Vande Woude GF. Proto-oncogene expression in germ cell development. Trends Genet 1988 ; 4 : 183-7.

20. Sagata $N$, Watanabe GF, Vande Woude GF, Ikawa Y. The c-mos protooncogene is a cytostatic factor responsible for meiotic arrest in vertebrate eggs. Nature $1989 ; 342$ : 512-8.

21. Soussi $T$, Caron de Fromentel C, Méchali M, May P, Kress M. Cloning and characterization of a cDNA from Xenopus laevis coding for a protein homologous to human and murine p53. Oncogene $1987 ; 1$ : 71-8. 
22. Mazabraud A, Wegnez $M$, Denis $H$. Origin of scveral abundant proteins of amphibian oocytes. J Mol Evol 1992 (sous presse).

23. Ortenzi C, Miceli C, Bradshaw RA, Luporini $P$. Identification and initial characterization of an autocrine pheromone receptor in the protozan ciliatc Euplotes raikovi. J Cell Biol 1990 ; 111: 607-14.

24. De Feo-Jones D, Scolnick EM, Koller $\mathrm{R}$, Dhar R. ras-related gene sequences identificd and isolated from Saccharomyces cerevisiae. Nature 1983 ; 306 : 707-9.

25. McCormick A, Campisi J. Cellular aging and senescence. Curr Opinion Cell Biol $1991 ; 3: 230-4$.

26. Bradley A, Evans $M$, Kaufman $\mathbf{M H}$, Robertson E. Formation of germ-line chimaeras from embryo-derived teratocarcinoma cell lines. Nature 1984 ; 309 : 255-6.

27. Tam LW, Kirk D. The program for cellular differentiation in Volvox carteri as revealed by molecular analysis of development in a gonidialess/somatic regenerator mutant. Development 1991; 112 : 571-80.

28. Strauss M, Griffin BE. Cellular immortalization : an essential step or merely a risk factor in DNA virus-induced transformation? Cancer Cells $1990 ; 2$ : 360-5.

29. Percira-Smith OM, Smith JR. Evidence for the recessive nature of cellular immortality. Science 1983 ; 221 : 964-6.

30. Shay JW, Wright WE, Werbin $H$ Defining the molecular mechanisms of human cell immortalization. Biochim Biophys Acta 1991; 1072 : 1-7.

31. Pereira-Smith OM, Smith JR. Genetic analysis of indefinite division in human cells : identification of four complementation groups. Proc Natl Acad Sci USA 1988 ; 85 : 6042-6.

32. Goldstein S. Replicative senescence : the human fibroblast comes of age. Science 1990 ; $249: 1129-33$

33. Olovnikov AM. A theory of marginotomy. J Theor Biol 1973; 41: 181-90.

34. Blackburn EH. Structure and function of telomeres. Nature 1991; 350 : 569-73.

35. Counter CM, Avilion AA, LeFeuvre $\mathrm{CE}$, et al. Telomere shortening associated with chromosome instability is arrested in immortal cells which express telomerase activity. EMBO J 1992 ; 11 : 1921-9.

36. Illmensee K, Mahowald AP. Transplantation of posterior polar plasm in Drosophila. Induction of germ cclls at the anterior pole of the egg. Proc Nall Acad Sci USA 1974 71 : 1016-20.

37. Mahowald AP. Germ plasm revisited and illuminated. Science $1992 ; 255$ : 1216-7.

38. Bounoure L. La lignće germinale chez les batraciens anoures. In : Wolff E, cd. L'Origine de la lignée germinale. Paris : Hermann, 1964: 205-34

39. Togashi S, Kobayashi S, Okada M Functions of maternal mRNA as a cytoplasmic factor responsible for pole ccll formation in Drosophila embryos. Dev Biol 1986 ; $118: 352-60$
40. Ephrussi A, Dickinson LK, Lehmann $\mathrm{R}$. oskar organizes the germ plasm and directs localization of the posterior determinant nanos. Cell $1991 ; 66$ : 37-50.

41. Hay B, Jan LY, Jan YN. A protein component of Drosophila polar granules is endoded by vasa and has cxtensive sequence similarity to ATP-dependent helicases. Cell 1988 ; 55 : 577-87.

42. Lasko PF, Ashburner M. The product of the Drosophila gene vasa is very similar to eukaryotic initiation factor-4A. Nature 1988 $335: 611-17$.

\section{Summary}

Origin of the germ line and of cell mortality

Early embryos of many animals set apart a small number of cells which make up the germ line. Germ cells are potentially immortal, in contrast to somatic cells which are mortal, i.e. able to carry out a finite number of divisions. We propose an evolutionary interpretation of cell mortality. We assume that this property was acquired by the most primitive animals and played a crucial role to limit the size of those rudimentary organisms. For the earliest animals, cell mortality was an innovation and hence a gain of functions. Therefore, mortal cells should have additional functions with respect to immortal or immortalized cells. Such a difference can be observed in some extant organisms, since several genes or gene products must be inactivated to convert a mortal cell into an immortal one.

\section{Remerciements}

Les auteurs remercient Maurice Wegnez, Janine Beisson, Bernard Mignotte, Alain Collenot et Isabelle Guénal pour leurs critiques et leurs conseils pendant la préparation du manuscrit de cet article. 